\title{
Profiling serum antibodies to Mycobacterium tuberculosis proteins in rhesus monkeys with nontuberculous Mycobacteria
}

\author{
Fangui MIN ${ }^{1,2)}$, Jinchun PAN ${ }^{1,2)}$, Ruike WU ${ }^{1,2)}$, Meiling $\mathrm{CHEN}^{1,2)}$, Huiwen $\mathrm{KUANG}^{1,2)}$, and \\ Weibo $\mathrm{ZHAO}^{1,2)}$ \\ 1) Guangdong Laboratory Animals Monitoring Institute, Guangzhou 510663, P.R. China \\ ${ }^{2)}$ Guangdong Provincial Key Laboratory of Laboratory Animals, Guangzhou 510663, P.R. China
}

\begin{abstract}
Recent evidence indicates that the prevalence of diseases caused by nontuberculous mycobacteria (NTM) has been increasing in both human and animals. In this study, antibody profiles of NTM in rhesus monkeys (Macaca mulatta) were determined and compared with those of monkeys infected with Mycobacterium tuberculosis complex (MTBC). Antibodies against $10 \mathrm{M}$. tuberculosis proteins, purified protein derivative (PPD), and mammalian old tuberculin (MOT) were detected in 14 monkeys naturally infected with NTM by indirect ELISA. Sera from 10 monkeys infected with MTBC and 10 healthy monkeys were set as controls. All antigens showed high serological reactivities to MTBC infections and low reactivities in healthy monkeys. NTM infections showed strong antibody responses to MOT and PPD; moderate antibody responses to $16 \mathrm{kDa}, \mathrm{U} 1, \mathrm{MPT} 64 \mathrm{~L}, 14 \mathrm{kDa}$, and TB16.3; and low antibody responses to 38kDa, Ag85b, CFP10, ESAT-6, and CFP10-ESAT-6. According to the criteria of MTBC, only CFP10, ESAT-6, and CFP10-ESAT-6 showed negative antibody responses in all NTM infections. Taken together, these results suggest that positive results of a PPD/MOT-based ELISA in combination with results of antibodies to M. tuberculosis-specific antigens, such as CFP10 and ESAT-6, could discriminate NTM and MTBC infections. Two positive results indicate an MTBC infection, and a negative result for an M. tuberculosis-specific antigen may preliminarily predict an NTM infection.
\end{abstract}

Key words: antibody, M. tuberculosis proteins, nontuberculous mycobacteria, rhesus monkeys

\section{Introduction}

Mycobacterium comprises more than 100 different species of rod-shaped bacteria. Most mycobacteria, except for Mycobacterium tuberculosis complex (MTBC) and $M$. leprae, are defined as nontuberculous mycobacteria (NTM) [13]. Unlike MTBC, more than half of NTM are not contagious, and the remaining NTM are always associated with opportunistic infections [1]. Both human and animals can be infected with NTM $[4,5]$. There are many reports on research into identification, diagnosis, and treatment of NTM in human patients, but few reports focused on those in nonhuman primates. A small number of case reports on nonhuman primate NTM demonstrated highly similar outcomes to M. tuberculosis infections in tuberculin skin test (TST) responsiveness, clinical symptoms, and necropsies [3, 15].

Because of similarities in symptoms to MTBC, diagnosing NTM is actually very difficult. The most used biochemical methods based on culture for species iden-

(Received 25 March 2015 / Accepted 25 June 2015 / Published online in J-STAGE 2 October 2015)

Address corresponding: W. Zhao, Guangdong Laboratory Animals Monitoring Institute, Guangzhou 510663, P.R. China

Authors' contributions: FM, JP, and WZ conceived and designed the study. FM, JP, and RW performed the experiments. FM wrote the manuscript.

All co-authors read and approved the final manuscript.

(C)2016 Japanese Association for Laboratory Animal Science 
tification are laborious and time-consuming, sometimes even giving false results [14]. Molecular methods are rapid and specific, but the efficiencies of sera/plasma-, feces-, nasal swab-based PCRs are always unsatisfactory. Previously, we established an indirect ELISA method for diagnosing nonhuman primate tuberculosis based on $10 \mathrm{M}$. tuberculosis proteins, purified protein derivative (PPD), and mammalian old tuberculin (MOT) [8]. After further optimization for detection procedures, the method was more stable and presented ideal cutoff values of $0.2-0.3\left(\mathrm{OD}_{450}\right.$ values). In this study, plasma antibodies to the above 12 antigens were determined by indirect ELISA to provide opportunities for research of NTM serodiagnosis in rhesus monkeys (Macaca mulatta).

\section{Materials and Methods}

\section{Animals and infection status}

In a breeding farm that had experienced outbreak of tuberculosis, positive TST reactions emerged continuously in a rhesus monkey colony during routine quarantines. Fourteen rhesus monkeys showing positive TST reactions at least once were necropsied and identified as having NTM infections by tissue-based PCR using two primer pairs consisting of $16 \mathrm{~S}$ rRNA and ESAT- 6 gene sequences to discriminate NTM and MTBC infections as previously reported but with some modifications [12]. Here, we detected the 300 bp bands of the ESAT- 6 gene with the following primers: F, 5'-ATGACAGAGCAGCAGTG-3'; R, 5'-GCCCTATGCGAACATCCC-3'. Gross and microscope observations were recorded and analyzed according to the rules of $M$. tuberculosis infected monkeys [9]. The experimental protocol was reviewed and approved by the Institutional Animal Care and Use Committee of Guangdong Laboratory Animals Monitoring Institute (AAALAC accredited).

\section{Sera}

Sera from NTM infections confirmed postmortem were used in this study. Ten positive control sera were from monkeys identified as having M. tuberculosis infections by necropsy and bacterial culture, which were used in our previous report [8]. Ten sera from healthy rhesus monkeys with negative TST reactions were set as negative controls. All sera were separated by centrifugation and stored at $-80^{\circ} \mathrm{C}$.

\section{Antibody detection by ELISA}

The ELISA procedure was performed as previously reported with some improvements of the reaction system [8]. Here, the coating concentration for all $10 \mathrm{M}$. tuberculosis proteins (CFP10-ESAT-6, ESAT-6, CFP10, $\mathrm{Ag} 85 \mathrm{~b}, \mathrm{MPT} 64 \mathrm{~L}, \mathrm{U} 1, \mathrm{~TB} 16.3,38 \mathrm{kDa}, 16 \mathrm{kDa}$, and $14 \mathrm{kDa}$ ) was $0.5 \mu \mathrm{g} / \mathrm{ml}$. The coating concentration for PPD and MOT was 4,000 IU/ml. Goat anti-monkey IgG antibody conjugated to horseradish peroxidase was changed to mouse anti-monkey IgG monoclonal antibody conjugated to horseradish peroxidase diluted $1: 20,000$. After further optimization for detection procedures, the method was more stable and presented ideal cutoff values of $0.2-0.3$ ( $\left(\mathrm{OD}_{450}\right.$ values), which are shown in Table 2.

\section{Data analysis}

All numerical parameters are presented as the mean \pm SD. A heat map was used to illustrate the antibody profiles.

\section{Results}

\section{Infectious outcomes of NTM}

The infectious outcomes are listed in Table 1. No obvious clinical symptoms except occasional cough were observed for 2 monkeys. Thirteen of the 14 monkeys presented gross or histopathological lesions in at least one site, and only 1 monkey showed no pathological changes at necropsy. Bronchial lymph nodes and lungs were most often included in the infections, indicating that NTM mainly spread via the respiratory route. The extrapulmonary organs, such as the spleen, liver, and kidney, were also involved in the infections, which demonstrated that NTM could result in systemic infections in monkeys.

\section{Detection of antibodies in NTM infections}

Serological responses to $10 \mathrm{M}$. tuberculosis antigens, PPD, and MOT were measured by indirect ELISA, and the OD values are listed in Table 2. Positive ratios were calculated according to the criteria of tuberculosis in nonhuman primates. The results showed that all monkeys presented positive antibody responses to PPD and MOT and negative antibody responses to CFP10, ESAT-6, and CFP10-ESAT-6. There was only one monkey that gave a positive antibody response to $\mathrm{Ag} 85 \mathrm{~b}$, with a positive ratio of $7.1 \%$. For the other antigens, the positive ratios 
Table 1. The outcomes of monkeys naturally infected with NTM

\begin{tabular}{lcl}
\hline \multicolumn{1}{c}{ Infectious outcomes } & Percentage of animals & Animal numbers \\
\hline Clinical results & & \\
Occasional cough & $11.5 \%(2 / 14)$ & 2,7 \\
TST-positive reaction ${ }^{1}$ & $100 \%(14 / 14)$ & All animals \\
Gross observation & & \\
$\quad$ Enlarged bronchial lymph nodes & $71.4 \%(10 / 14)$ & $2,3,5-9,11-13$ \\
Granuloma in lung & $78.6 \%(11 / 14)$ & $1,2,3,5-9,11-13$ \\
Granuloma in spleen & $42.9 \%(6 / 14)$ & $2,3,5-7,10$ \\
Granuloma in liver & $28.6 \%(4 / 14)$ & $2,5,7,10$ \\
Granuloma in kidney & $11.5 \%(2 / 14)$ & 7,10 \\
Histopathology & & \\
Bronchial lymph nodes & $71.4 \%(10 / 14)$ & $2,3,5-9,11-13$ \\
Lung & $85.7 \%(12 / 14)$ & $1-3,5-13$ \\
Spleen & $42.9 \%(6 / 14)$ & $2,3,5-7,10$ \\
Liver & $28.6 \%(4 / 14)$ & $2,5,7,10$ \\
Kidney & $11.5 \%(2 / 14)$ & 7,10 \\
\hline
\end{tabular}

${ }^{1}$ There was at least one positive reaction after receiving repeated TSTs. ${ }^{2}$ The following findings were considered specific pathological changes: caseous granulomas, nonnecrotic granulomas, granulomatous inflammation, etc. ${ }^{3}$ Animal numbers: 1, H01025; 2, S03092; 3, G06031; 4, G10104; 5, no ID (old); 6, S01066; 7, G10130; 8, G07284; 9, S01220; 10, S01732; 11, G09088; 12, G09109; 13, G09025;14, no ID (young).

Table 2. Antibody responses to MOT, PPD, and M. tuberculosis-specific proteins

\begin{tabular}{lccrl}
\hline \multicolumn{1}{c}{ Antigens } & OD $_{450}$ values & Cutoff values & Positive ratios & Positive animal numbers \\
\hline MOT & $1.54 \pm 0.60$ & 0.280 & $100 \%(14 / 14)$ & All animals \\
PPD & $1.62 \pm 0.70$ & 0.217 & $100 \%(14 / 14)$ & All animals \\
16kDa & $0.52 \pm 0.44$ & 0.257 & $42.9 \%(6 / 14)$ & $1-3,6-8$ \\
U1 & $0.66 \pm 0.62$ & 0.320 & $50.0 \%(7 / 14)$ & $1-3,6,7,11,13$ \\
MPT64L & $0.38 \pm 0.31$ & 0.246 & $50.0 \%(7 / 14)$ & $1-3,6,7,11,13$ \\
14kDa & $0.38 \pm 0.29$ & 0.286 & $42.9 \%(6 / 14)$ & $1-3,6,7,11$ \\
TB16.3 & $0.35 \pm 0.32$ & 0.271 & $28.6 \%(4 / 14)$ & $1,3,6,11$ \\
38kDa & $0.22 \pm 0.17$ & 0.262 & $14.3 \%(2 / 14)$ & 1,2 \\
Ag85b & $0.16 \pm 0.07$ & 0.248 & $7.1 \%(1 / 14)$ & 1 \\
CFP10 & $0.18 \pm 0.08$ & 0.259 & $0(0 / 14)$ & None \\
ESAT-6 & $0.13 \pm 0.05$ & 0.214 & $0(0 / 14)$ & None \\
CFP10-ESAT-6 & $0.16 \pm 0.04$ & 0.229 & $0(0 / 14)$ & None \\
\hline
\end{tabular}

for the antibodies against them ranged from 14.3 to $50 \%$.

\section{Antibody profiles}

According to the OD values of each selected antigen, a heat map based on color intensity shifting from red to yellow to green to blue was generated for the relative strength of reactivity of each antigen in individual sera from high to low levels (Fig. 1). For NTM infections, three major antigen clusters emerged in multivariate analysis of the profiles of antibodies against all 12 antigens. MOT and PPD were included in cluster 1 with the highest reactivities, followed by $16 \mathrm{kDa}, \mathrm{U} 1, \mathrm{MPT} 64 \mathrm{~L}$, $14 \mathrm{kDa}$, and TB16.3 in cluster 2, while $38 \mathrm{kDa}, \mathrm{Ag} 85 \mathrm{~b}$, CFP10, ESAT-6, and CFP10-ESAT-6 were classified into clusters 3 , which showed the lowest reactivities.
Different from NTM infections, the MTBC infections gave no obvious clusters for any antigens. Each infection presented positive antibody responses to more than half of the antigens. But no single antigen reached $100 \%$ positive serological reactivity in MTBC infections. The healthy monkeys showed negative antibody responses to all antigens, exhibiting the 2 lowest of colors on the heat map.

\section{Comparisons of antibody characteristics between NTM} and MTBC infections

Antibodies against the 12 antigens in 10 tuberculosispositive monkeys and 10 healthy monkeys were compared with those of monkeys with NTM infections. All antigens showed high serological reactivities in most 


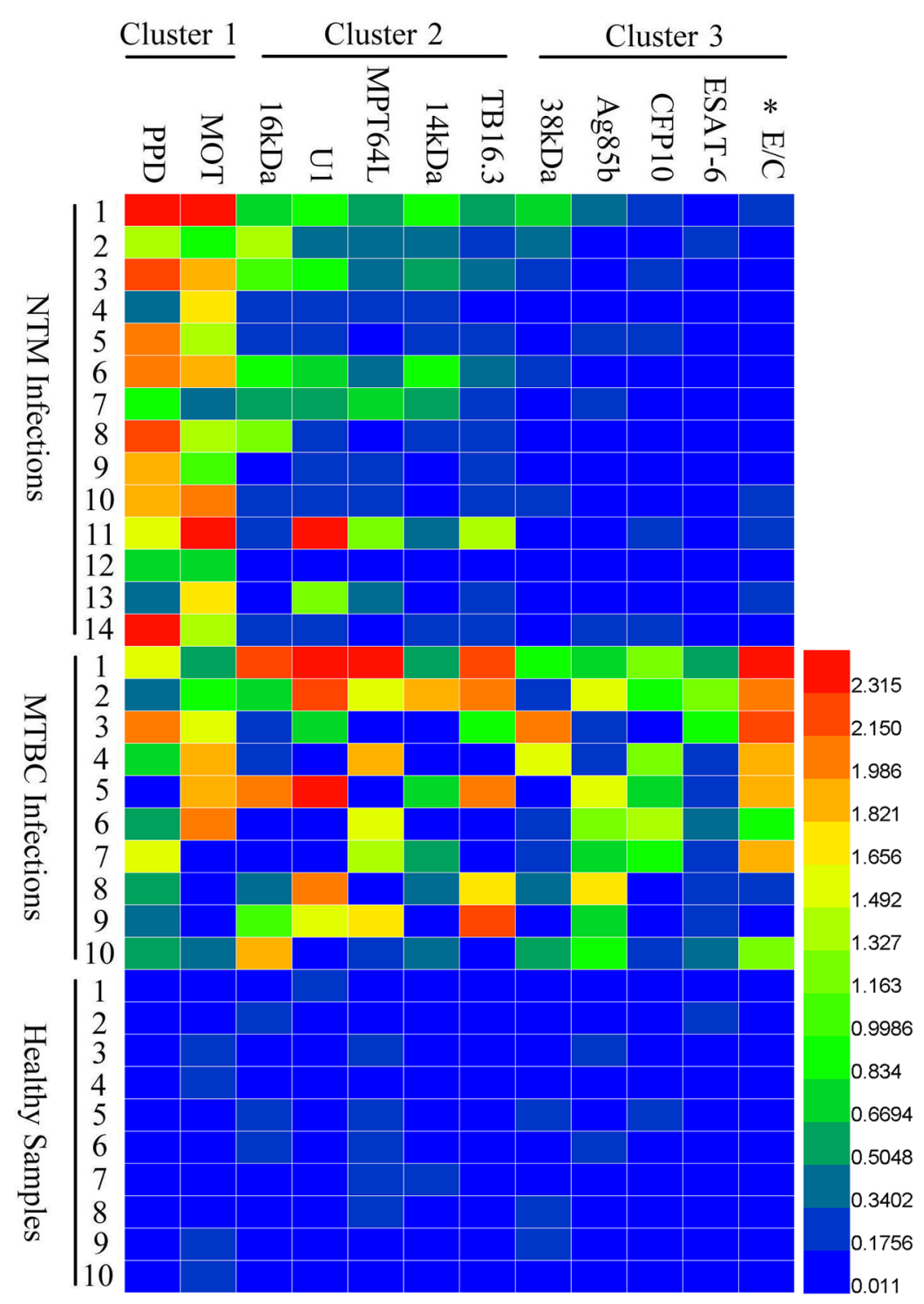

Fig.1. Heat map of antibody reactivity to 12 antigens. Selected antigens are listed at the top of the heat map and divided into 3 clusters according to the relative strength of antibody reactivity of each antigen in sera from monkeys with NTM infections. Sera are numbered on the left side of the heat map. The color intensity, which ranges from red to yellow to green to blue, indicates the reactivity from the high to low levels. *E/C=CFP10-ESAT-6.

monkeys with tuberculosis infections and low reactivities in healthy animals. The antibody levels (mean \pm SD) in monkeys with tuberculosis tended to be higher than those in healthy monkeys for all antigens, while monkeys with NTM presented different antibody characteristics. Antibodies for PPD and MOT in monkeys with NTM showed the same characteristics as monkeys with tuberculosis, demonstrating higher antibody levels than in healthy monkeys. For the other antigens, the levels of antibodies against them were lower than in the monkeys with tuberculosis. No statistical differences between monkeys with NTM and healthy monkeys were found in antibodies to CFP10-ESAT-6, ESAT-6, CFP10, Ag85b, and $38 \mathrm{kDa}$. For the remaining antigens, MPT64L, U1, TB16.3, 16kDa, and $14 \mathrm{kDa}$, antibodies to them were higher in monkeys with NTM than those in healthy monkeys. 


\section{Discussion}

Tuberculosis in nonhuman primates is highly contagious disease, and the most important causative agent is M. tuberculosis. However, in recent years, NTM in nonhuman primates has also been identified in the etiology of tuberculosis-like syndromes [3, 15]. For human patients, especially those following initiation of antiretroviral treatment, NTM is often underdiagnosed or misdiagnosed as tuberculosis [10]. Generally, an accurate diagnosis can be made for patients in whom NTM is isolated from one or more sources. However, it is difficult to diagnose patients in whom NTM has not been isolated, and it is also a challenge to differentiate NTM infection from MTBC infection. Therefore, sequencing of the 16SrRNA genes is suitable for identification of bacteria [6], which is limited by DNA sources of clinical samples.

For most nonhuman primates breeding farms in China, tuberculosis screening at routine quarantines is mainly based on PPD- and MOT-TSTs. In a breeding farm that had battled an outbreak of tuberculosis, we found 14 rhesus monkeys with a tuberculosis-like disease, showing positive TST reactions and typical lesions (Table 1). Further PCR-based analysis proved that they had NTM infections. In this study, we extensively used serum samples to profile the antibodies against $12 \mathrm{M}$. tuberculosis antigens, providing hope for the research of NTM serodiagnosis.

By using indirect ELISA, we detected the antibody responses to 10 recombinant $M$. tuberculosis proteins, PPD, and MOT in 14 monkeys with NTM infections. According to the cutoff values of each antigen for diagnosing tuberculosis infection, the percentages of animals positive for antibodies against the different antigens were determined. The highest ratios of positive antibody responses were found for MOT and PPD, both of which were $100 \%$, followed by $16 \mathrm{kDa}, \mathrm{U} 1$, MPT $64 \mathrm{~L}, 14 \mathrm{kDa}$, TB16.3, 38kDa, and Ag85b, the ratios of which ranged from 7.1 to $50 \%$. However, all animals showed negative antibody responses for CFP10, ESAT-6, and CFP10ESAT-6.

The antibody information (OD values) was further used to draw a heat map, which classified the antigens into 3 clusters according to the relative strength of antibody reactivity in monkeys with NTM infections. MOT and PPD showed the highest antibody reactivities, followed by $16 \mathrm{kDa}$, U1, MPT64L, 14kDa, and TB16.3. The remaining 5 antigens presented low antibody reactivities. The healthy monkeys showed negative antibody responses to all antigens with the 2 lowest classes of colors on the heat map. Different from the monkeys with NTM infections, each monkey with an MTBC infection presented positive antibody responses to more than half of the antigens, giving no obvious clusters for all antigens. The monkeys with MTBC infections had not yet reached $100 \%$ positive antibody responses to any single antigen, though combination of the results for all antigens resulted in a $100 \%$ detection rate. There are two possible reasons for this result: (1) the lack of performance of a single-antigen ELISA and (2) false negative antibody responses possibly caused by serum protein degradation as a result of preservation of samples for too long. The MTBC-positive sera used in this study were collected before 2011 .

In our previous study regarding diagnosis of nonhuman primate tuberculosis, CFP10 and Ag85b showed the highest diagnostic potential, followed by ESAT- 6 and CFP10-ESAT-6 [8]. As we have optimized the ELISA system, the previous data could not be used directly for comparison with NTM infections. Here, we examined 10 naturally tuberculosis-positive sera and 10 sera from healthy monkeys by the optimized method, and the antibody characteristics of the positive sera were the same as those in our previous data, with significantly higher levels in the positive sera than in the negative sera. When compared with the levels in the negative sera, the levels of antibodies against PPD, MOT, 16kDa, U1, MPT64L, $14 \mathrm{kDa}$, and TB16.3 in sera from monkeys with NTM infections tended to be higher, indicating that these antigens could not distinguish the NTM and MTBC infections, though the levels of antibodies to MPT64L and TB16.3 in sera from monkeys with NTM infections were lower than those in sera from monkeys with tuberculosis infections. Due to moderate diagnostic potential for tuberculosis, $38 \mathrm{kDa}$ antigen was also not suitable for distinguishing NTM and MTBC infections; even its antibody reactivity in sera from monkeys with NTM infections was low. There were no differences between sera from monkeys with NTM infections and sera from healthy monkeys with respect to for Ag85b, CFP10, ESAT-6, and CFP10-ESAT-6, while significant differences were found between sera from monkeys with NTM and tuberculosis infections, suggesting their great values in discriminating NTM and MTBC infections. Though there are a few reports indicating the immunological 
cross-reactivity of $M$. tuberculosis ESAT-6 with ESAT-6 homologues of $M$. avium and M. kansasii in an IFN- $\gamma$ assay $[2,11]$, no evidences was found indicating that serum antibodies to ESAT- 6 could be detected in NTM infections.

Taken together, these results suggest that positive results of a PPD/MOT-based TST or serum ELISA in combination with results of antibodies for M. tuberculosis-specific antigens, such as CFP10 and ESAT-6, could discriminate NTM and MTBC infections. Two positive results indicate an MTBC infection, and a negative result for an M. tuberculosis-specific antigen may preliminarily predict but not confirm an NTM infection, as the sensitivity of the M. tuberculosis-specific antigen-ELISA is not $100 \%$ [7]. Our findings in this study, although preliminary in nature due to the small clinical sample sizes, contribute knowledge to the ongoing development of serodiagnosis of mycobacteria infection. Further studies will employ a larger number of specimens to validate the diagnostic value of specific antigens.

\section{Conflict of interest}

The authors declare that they have no competing interests.

\section{Acknowledgments}

This work was supported by grants 2013B020307005 and 2015A030302029 from the Guangdong Provincial Science \& Technology Project.

\section{References}

1. Holland, S.M. 2001. Nontuberculous mycobacteria. Am. J. Med. Sci. 321: 49-55. [Medline] [CrossRef]

2. Hur, Y.G., Crampin, A.C., Chisambo, C., Kanyika, J., Houben, R., Ndhlovu, R., Mzembe, T., Lalor, M.K., Saul, J., Branson, K., Stanley, C., Ngwira, B., French, N., Ottenhoff, T.H., Dockrell, H.M., and Gorak-Stolinska, P. 2014. Identification of immunological biomarkers which may differentiate latent tuberculosis from exposure to environmental nontuberculous mycobacteria in children. Clin. Vaccine Immunol. 21: 133-142. [Medline] [CrossRef]

3. Jackson, R.K., Juras, R.A., Stiefel, S.M., and Hall, J.E. 1989. Mycobacterium kansasii in a rhesus monkey. Lab. Anim. Sci. 39: 425-428. [Medline]
4. Kankya, C., Muwonge, A., Djønne, B., Munyeme, M., Opuda-Asibo, J., Skjerve, E., Oloya, J., Edvardsen, V., and Johansen, T.B. 2011. Isolation of non-tuberculous mycobacteria from pastoral ecosystems of Uganda: public health significance. BMC Public Health 11: 320. [Medline] [CrossRef]

5. Malama, S., Munyeme, M., Mwanza, S., and Muma, J.B. 2014. Isolation and characterization of non tuberculous mycobacteria from humans and animals in Namwala District of Zambia. BMC Res. Notes 7: 622. [Medline] [CrossRef]

6. Janda, J.M. and Abbott, S.L. 2007. 16S rRNA gene sequencing for bacterial identification in the diagnostic laboratory: pluses, perils, and pitfalls. J. Clin. Microbiol. 45: 27612764. [Medline] [CrossRef]

7. Min, F.G., Wang, J., and Zhang, Y. 2015. Performance of ESAT-6 for serodiagnosis of nonhuman primate tuberculosis: a meta analysis. J. Adv. Vet. Anim. Res. 2: 107-114. [CrossRef]

8. Min, F., Zhang, Y., Huang, R., Li, W., Wu, Y., Pan, J., Zhao, W., and Liu, X. 2011. Serum antibody responses to 10 Mycobacterium tuberculosis proteins, purified protein derivative, and old tuberculin in natural and experimental tuberculosis in rhesus monkeys. Clin. Vaccine Immunol. 18: 2154-2160. [Medline] [CrossRef]

9. Min, F., Zhang, Y., Pan, J., Wang, J., and Yuan, W. 2013. Mycobacterium tuberculosis infection in rhesus monkeys (Macaca mulatta) and evaluation of ESAT-6 and CFP10 as immunodiagnostic antigens. Exp. Anim. 62: 281-293. [Medline] [CrossRef]

10. Mogambery, J.C., Motala, A., Padayachee, K., Jozi, C., and Dawood, H. 2014. Nontuberculous mycobacteria immune reconstitution syndrome. Case Rep. Med. 2014: 964612. [Medline]

11. Parsons, S.D., de Villiers, C., Gey van Pittius, N.C., Warren, R.M., and van Helden, P.D. 2010. Detection of Mycobacterium kansasii infection in a rhesus macaque (Macaca mulatta) using a modified QuantiFERON-TB Gold assay. Vet. Immunol. Immunopathol. 136: 330-334. [Medline] [CrossRef]

12. Singh, S., Gopinath, K., Shahdad, S., Kaur, M., Singh, B., and Sharma, P. 2007. Nontuberculous mycobacterial infections in Indian AIDS patients detected by a novel set of ESAT-6 polymerase chain reaction primers. Jpn. J. Infect. Dis. 60: 14-18. [Medline]

13. Tortoli, E. 2006. The new mycobacteria: an update. FEMS Immunol. Med. Microbiol. 48: 159-178. [Medline] [CrossRef]

14. van Ingen, J., Boeree, M.J., Dekhuijzen, P.N., and van Soolingen, D. 2009. Environmental sources of rapid growing nontuberculous mycobacteria causing disease in humans. Clin. Microbiol. Infect. 15: 888-893. [Medline] [CrossRef]

15. Wachtman, L.M., Miller, A.D., Xia, D., Curran, E.H., and Mansfield, K.G. 2011. Colonization with nontuberculous mycobacteria is associated with positive tuberculin skin test reactions in the common marmoset (Callithrix jacchus). Comp. Med. 61: 278-284. [Medline] 